\title{
OBSERVATIONS ON INTRAVENTRICULAR HEMICHOLINIUM-3-INDUCED EEG SEIZURES*†
}

\author{
M. K. Shellenberger $\ddagger$ and Edward F. Domino \\ Department of Pharmacology, University of Michigan, Ann Arbor, Michigan
}

(Accepted 9 February 1967)

\begin{abstract}
Summary-It was observed that intraventricular (i.vt.) injection of HC-3 produced markedly different effects upon the EEG of the dog, dependent upon dose. The high dose $(5 \mathrm{mg})$ investigated in this paper produced seizures with a 20 min latency. This effect was compared with the effects of several drugs known to act on the cholinergic system, and in addition the effect on acetylcholinesterase (AChE) of several brain regions was determined. It was concluded that the HC-3-induced EEG seizures probably did not occur as a result of $\mathrm{AChE}$ inhibition but resulted from a direct action on possible cholinergic receptors. Suggestions for the nature of this action are discussed.
\end{abstract}

\section{INTRODUCTION}

HemicholiniUm-3 (HC-3) has been both an exciting and disappointing pharmacological tool for those interested in central cholinergic mechanisms. Perhaps its greatest limitation is the difficulty with which it enters the brain (DOMER and SCHUELER, 1960) or consistently affects the central nervous system, even with large parenteral doses (DREN and DoMINo, 1965). Although there are reports on the direct central actions of $\mathrm{HC}-3$ given parenterally (SCHUELER, 1955; KASE and BORISON, 1958 and others) these effects are not widely accepted. The peripheral actions of HC-3 mask any central action the drug may have.

In order to observe consistently the effects of HC-3 on the brain it is necessary to bypass the blood-brain barrier by injecting the drug either into the substance or ventricles of the brain. HeBB et al. (1964) injected HC-3 directly into the caudate nucleus of rats and found a marked and rapid (within minutes) reduction in acetylcholine (ACh) content and synthesis with little effect upon dopamine content. DREN and Domino (1965) injected HC-3 into the lateral ventricle of the paralyzed dog and found that the EEG of neocortical as well as subcortical structures was altered after approximately $1 \cdot 5-2 \cdot 5 \mathrm{hr}$. Recently these investigators have reported that the EEG slowing was associated with a decrease in the ACh content of periventricular structures (DREN and Domino, 1966) over a wide dose range of $\mathrm{HC}-3$.

Large doses of HC-3, given intraventricularly (i.vt.) cause subcortical EEG seizures, which may spread to the cortex, prior to the time that the high voltage slow waves appear. This indicates that, as in peripheral tissues (SCHUELER, 1960), HC-3 has varying actions, dependent upon dose. If this agent is to be a useful tool for the investigation of central

*Preliminary report given at ASPET meetings in Mexico City in July, 1966.

†Supported in part by PHS Grant NB-1311.

†Supported by NINDB Fellowship 1 F2 NB 29,637-01. 
cholinergic pathways, it becomes necessary to determine all of the possible cerebral actions of this drug upon i.vt. administration.

In addition to its inhibitory effect on choline transport (GARDINER, 1961; VANDER, 1962), HC-3 is reported to have both an anticholinesterase and a direct, curare-like action on the neuromuscular junction (SCHUELER, 1960). This paper describes the in vivo effects of HC-3 on acetylcholinesterase (AChE) in periventricular areas compared with the potent cholinesterase inhibitor, neostigmine. In addition, the effects of $\mathrm{HC}-3$ on subcortical and cortical EEG have been compared with those agents which are classified as $m$ and $n$ cholinergic antagonists. An attempt has been made to characterize the nature of HC-3-elicited EEG seizures. This preliminary study suggests they are not the result of cholinesterase inhibition, but rather are due to a direct action on receptors which may be cholinergic in nature.

\section{METHODS}

EEG studies were performed in beagle-like mongrel dogs of either sex weighing from 8-12 kg. The animals were paralyzed with decamethonium, intubated and artificially respired as described previously (Domino et al. 1967). The surgical procedures, which included placement of electrodes and cannulation of the femoral vein and artery, were performed under $70 \%$ nitrous oxide, $30 \%$ oxygen anesthesia. Subsequently, surgical sites were infiltrated with lidocaine $(1 \%)$ plus epinephrine. The animals were thus maintained in a pain-free, immobilized state.

Electrodes were stereotaxically placed in cortical areas $\operatorname{PrC}_{2}$ and $\mathrm{O}_{1}$, dorsal hippocampus and the caudate nucleus on the left side of the brain, according to the dog brain atlases of Lim et al. (1960), and AdRIANOV and Mering (1964). Monopolar recordings were made, with reference to an indifferent electrode placed in the nasion, using the Offner type $R$ Dynograph. The drugs listed in Table 1 were injected (i.vt.) through a needle placed into the left lateral ventricle above the dorsal hippocampus. The drugs were in $0.9 \%$ saline solution and injected in a volume of $0.2 \mathrm{ml}$ following the withdrawal of 0.3 to $0.4 \mathrm{ml}$ of cerebrospinal fluid (csf). The needle was flushed with csf following the drug injection in such a manner that no change in ventricular volume occurred.

TABLE 1. DRUGS given BY INTRAVENTricular RoUte

\begin{tabular}{|c|c|c|c|}
\hline Drug* & Dose in $\mathrm{mg}$ & $\begin{array}{l}\text { Molarity compared } \\
\text { to } 5.0 \mathrm{mg} \mathrm{HC}-3\end{array}$ & No. of animals \\
\hline $0.9 \% \mathrm{NaCl}$ & - & - & 3 \\
\hline $\mathrm{NaBr}$ & $2 \cdot 5$ & $\times 2$ & 3 \\
\hline Hemicholinium-3 bromide (HC-3) & $5 \cdot 00$ & - & 9 \\
\hline Neostigmine bromide (NEO). & $\begin{array}{l}2 \cdot 67 \\
0.0267\end{array}$ & $\begin{array}{l}\text { Equal } \\
\times 0.01\end{array}$ & $\begin{array}{l}4 \\
3\end{array}$ \\
\hline Tetramethylammonium chloride (TMA) & $1 \cdot 78$ & $\times 2$ & 3 \\
\hline Hexamethonium chloride $\left(\mathrm{C}_{\ell}\right)$ & $2 \cdot 45$ & Equal & 3 \\
\hline Atropine sulfate (ATRO.) & 6.96 & $\times 2$ & 6 \\
\hline $\begin{array}{l}\text { Atropine methyl nitrate } \\
\text { Atropine methyl iodide }\end{array}$ & $7 \cdot 30$ & $\times 2$ & 6 \\
\hline d-Tubocurarine chloride ( $d$-TUBO.) & $8 \cdot 35$ & Equal & 3 \\
\hline
\end{tabular}

*All drugs put into saline solution 
The animals were sacrificed by air injection at the time of development of a maximal pharmacological response, if one occurred. The head of each dog was rapidly cut in two, samples of brain taken for later AChE assay and immediately frozen in dry ice. At this time the location of the subcortical electrodes could be verified by gross observation. The areas sampled were: left caudate, thalamus, amygdala, hippocampus, sensory-motor and occipital cortex, as well as midline reticular formation ventral to the floor of the 4 th ventricle. The cholinesterase assay was performed by the titrimetric method described by JENSENHoLm et al. (1959) using acetyl- $\beta$-methacholine $\left(5 \times 10^{-3} \mathrm{M}\right)$, as substrate to provide greater specificity for AChE.

\section{RESULTS}

\section{Comparative effects of i.vt. HC-3 and neostigmine}

The progression of EEG effects following $5 \mathrm{mg}$ of HC-3 can be seen in Fig. 1. Approximately 20 min after injection, spiking appeared in the subcortical leads. The site of origin of these spikes is subcortical, but the precise location is unclear as the spikes occasionally appeared simultaneously in both the caudate nucleus and hippocampus, and at other times began first in the caudate nucleus. In this report, it can be seen that 20 min after
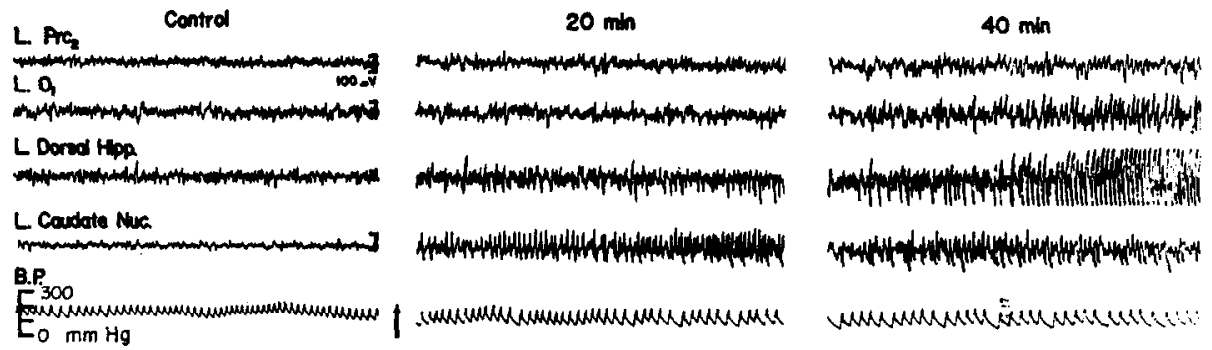

HC-3 (5.0 mg i.vt.)

$45 \mathrm{~min}$

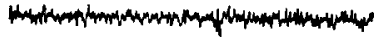

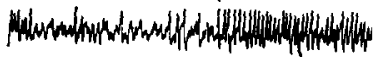

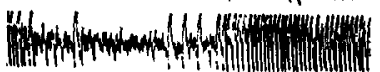

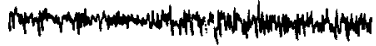

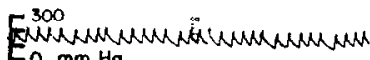

ENWWwh
$50 \min$
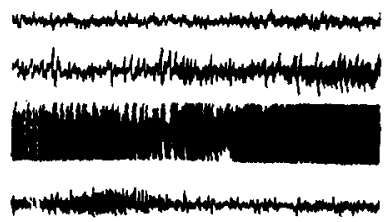

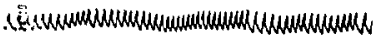

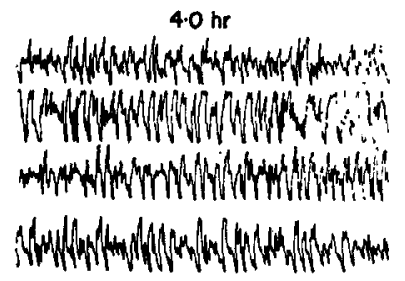

$2 \sec$

FIG. 1. EEG effects of a large dose of HC-3 given intraventricularly. Hemicholinium was given in a dose of $5 \mathrm{mg}$ into the left ventricle. The animal was locally anesthetized and on artificial ventilation. Segments of the EEG record were taken before and at various times following $\mathrm{HC}-3$, as indicated. Note that characteristic spike-like activity beginning in the caudate nucleus and hippocampus progressively increased in amplitude with the development of EEG seizures, particularly in the subcortical structures but projecting to the visual cortex as well. After $4 \mathrm{hr}$ the characteristic high voltage, slow wave pattern was seen diffusely. All recordings are monopolar. Symbols: L.-left side; $\mathrm{PrC}_{2}-$ precruciate cortex; $\mathrm{O}_{1}-\mathrm{Occi-}$ pital cortex; Dorsal Hipp-dorsal hippocampus; Caudate Nuc.-caudate nucleus; B. P.arterial blood pressure in $\mathrm{mm} \mathrm{Hg}$. Time base and voltage calibrations are as indicated. These symbols apply to all subsequent figures. 
injection, EEG spiking in the caudate nucleus is much better developed than in the hippocampus. The spiking developed into seizures of relatively low frequency which spread to the occipital cortical lead. After $45 \mathrm{~min}$, regardless of seizure origin, the hippocampus had the dominant activity and drove both the occipital cortex and caudate until the frequency of discharges became too great for the latter to follow. It was interesting to note that seizure discharge did not spread to the motor cortex. Most of the EEG leads were characterized by bursts of seizure activity of the type shown in the 40 to $50 \mathrm{~min}$ sequence until 2.5 to $3.0 \mathrm{hr}$ after injection. At this time the EEG showed slow waves of the type previously reported by DREN and Domino $(1965,1966)$, as shown in the $4 \mathrm{hr}$ segment of record in Fig. 1.

Figure 2 illustrates the development of the response to an equimolar dose ( $2.67 \mathrm{mg}$ i.vt.) of neostigmine. The immediate postinjection phase was invariably characterized by constant theta-like activity in the hippocampus. In 15-20 min this gave way to bursts of high voltage, high frequency activity which lasted 10-15 min. The EEG then showed a pattern of multiple spikes in the hippocampus and occipital cortex with slower high voltage activity in caudate and motor cortex shown in the 1-hr panel in Fig. 2.

The seizure activity caused by HC-3 became maximal within $30 \mathrm{~min}$ to $1 \mathrm{hr}$ after injection. It was felt that this latter time would be appropriate for determining the AChE activity of various periventricular and neocortical structures. Accordingly, animals were
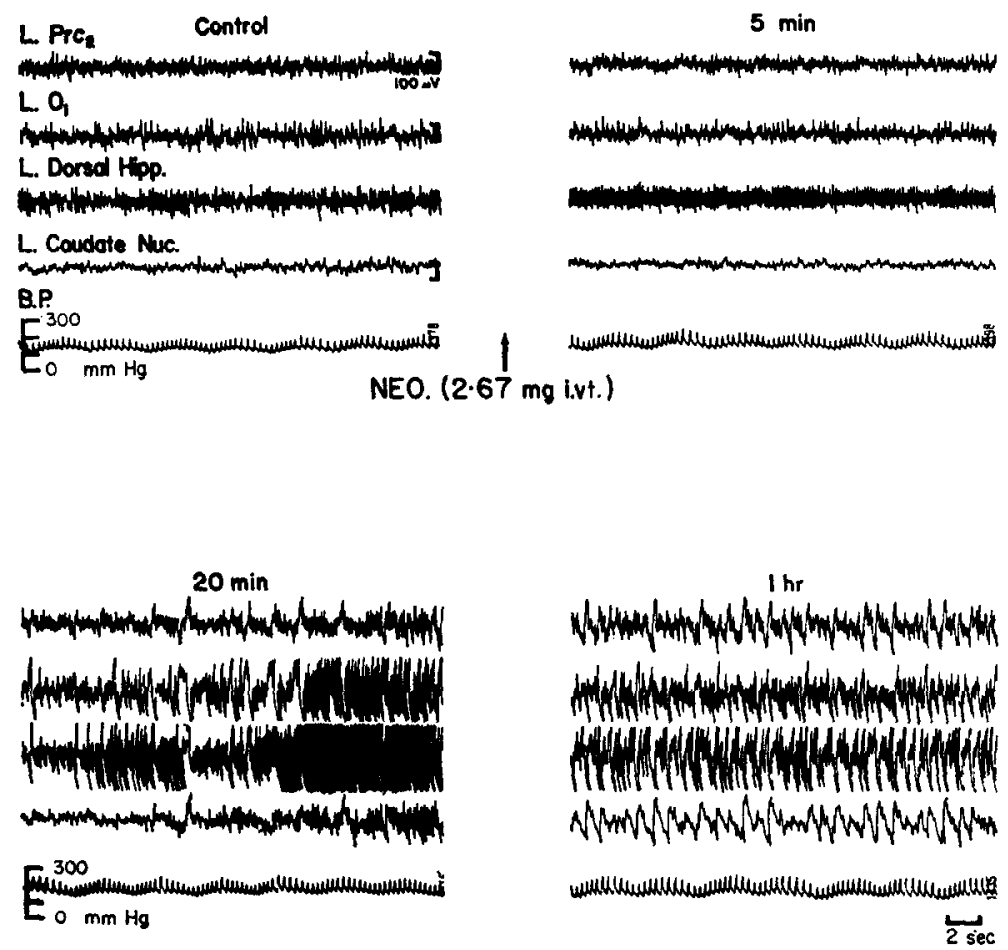

Fig. 2. EEG effects of neostigmine given intraventricularly. The effect of neostigmine in a dose equimolar to $5 \mathrm{mg}$ of HC-3 is illustrated. Note that at 20 min marked EEG seizures are evident in the dorsal hippocampus, caudate nucleus and occipital cortex. This seizure activity continues but at a slower frequency $1 \mathrm{hr}$ later. Note that the EEG effects of neostigmine are quite different from those of HC-3. 
sacrificed $1 \mathrm{hr}$ after the i.vt. injection of $\mathrm{HC}-3$, equimolar neostigmine $(2.67 \mathrm{mg}$ or 0.01 equimolar neostigmine, $26.7 \mu \mathrm{g}$ ), and the appropriate brain areas removed for assay. The latter dose was included because it is equimolar with the $50 \mu \mathrm{g}$ dose of HC-3, which has been shown to cause EEG slowing and reduction of ACh levels comparable to the $5 \mathrm{mg}$ dose (DREN and Domino, 1966). The mean $\pm \mathrm{SE}$ of AChE activity of the various brain areas for groups of 3-9 animals are shown in Fig. 3.

It can be seen that $5 \mathrm{mg}$ of $\mathrm{HC}-3$ injected i.vt. caused no measurable inhibition of AChE at $1 \mathrm{hr}$ when the seizure activity was maximal. On the other hand, neostigmine, in an equimolar dose, caused marked AChE inhibition in both subcortical and cortical tissues and significant inhibition in subcortical areas at the 0.01 equimolar dose.

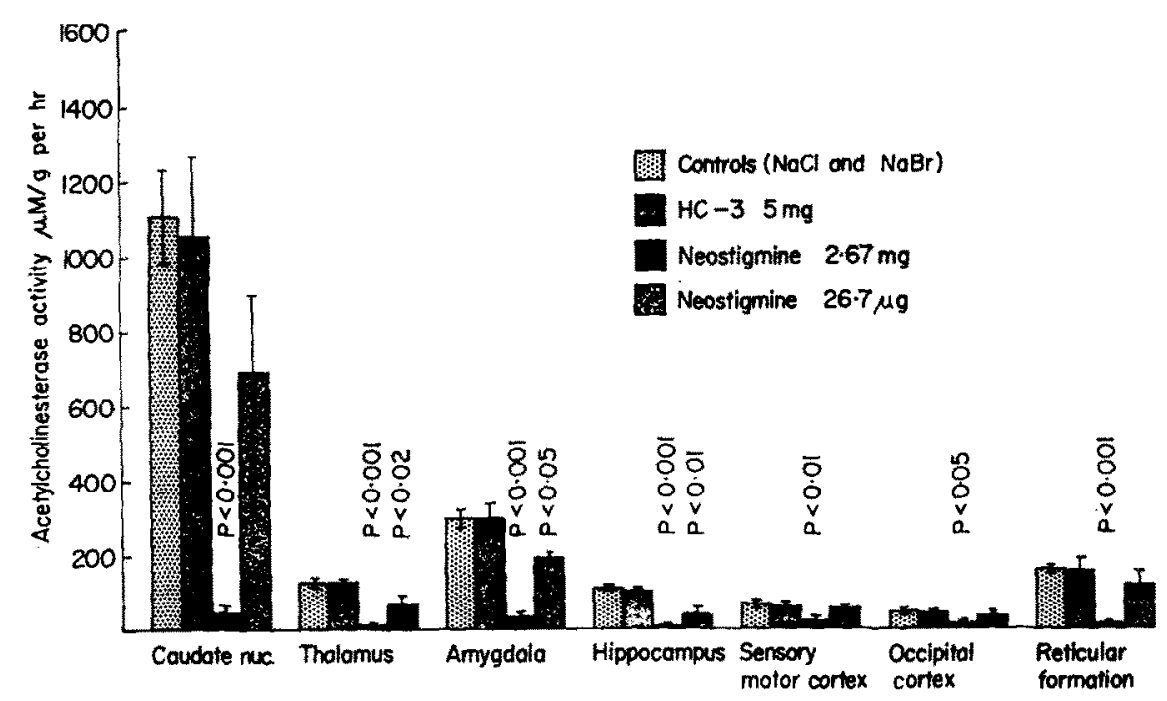

FIG. 3. Effects of $\mathrm{HC}-3$ and neostigmine on brain acetylcholinesterase. AChE activity is expressed as $\mu \mathrm{M}$ of acetyl- $\beta$ methacholine hydrolysed per $\mathrm{g}$ of tissue per $\mathrm{hr}$. The AChE activity of the caudate nucleus, medial thalamus, amygdala, hippocampus, motor cortex, occipital cortex and reticular formation is indicated by the height of the bars. Control brain AChE activity is indicated after $0.9 \%$ sodium chloride and/or equimolar sodium bromide. The effects of large equimolar doses of $\mathrm{HC}-3$ and neostigmine were compared to 0.01 as much neostigmine. The standard deviations of the mean (S.E.) are indicated by the small vertical lines. The probabilities (student " $t$ " test, group comparison) are given when significant.

\section{Comparative effects of various drugs containing quaternary nitrogens}

It was possible that the seizure activity of $\mathrm{HC}-3$ could be a relatively nonspecific ionic effect of the quaternary nitrogens. Therefore, the EEG effects of several quaternary compounds were compared with those of HC-3. Tetramethylammonium (TMA), the simplest of all quaternary nitrogen compounds, was administered in a twice equimolar dose $(1.78 \mathrm{mg}$ i.vt.) to obtain the same number of quaternary nitrogens as in HC-3. As shown in Fig. 4, TMA had no effect on either the cortical or subcortical EEG. The bis-quaternary compound hexamethonium, with a short six-carbon chain separating the cationic nitrogen structures, caused no generalized seizures but did result in spiking in the hippocampus when given in a dose $(2.45 \mathrm{mg})$ which was equimolar to $5 \mathrm{mg}$ of HC-3. 
Administration of the bis-quaternary compound $d$-tubocurarine ( $8.35 \mathrm{mg}$ i.vt.), resulted in almost immediate seizure discharge, as has been reported previously (FELDBERG et al., 1956). These seizures began subcortically and spread readily to the cortex.

The increased $n$ cholinergic effects that quaternization of atropine produces also facilitated EEG seizures, (Fig. 5). The tertiary base atropine and its quaternary analogue, methylatropine, were administered i.vt. in twice equimolar doses $(6.96$ and $7.3 \mathrm{mg}$, respectively), to provide a number of quaternary or tertiary structures equal to those in HC-3. The first effect of atropine was a reduction of the amplitude of discharge in the hippocampus and caudate nucleus which may have been produced by the resulting extreme hypertension. This was followed by a gradual increase in the voltage of all leads at $25 \mathrm{~min}$ with a pattern greatly resembling sleep and including spindles in the motor cortex. By $1 \mathrm{hr}$ the EEG pattern showed spikes in the hippocamus and to a less extent in the occipital cortex; however, no EEG seizures were observed.

Injection of methylatropine resulted in spiking within $5 \mathrm{~min}$, and seizures within 15-min which seemed to originate subcortically and then spread to the cortex. This was in contrast to HC-3-induced EEG seizures which did not spread to the cortex in the doses used.

All groups of animals were sacrificed, the pertinent brain areas assayed for AChE activity, and compared to $\mathrm{HC}-3$ and neostigmine. Except for neostigmine, none of the compounds caused significant inhibition although atropine caused inhibition bordering on significance in the hippocampus with the large equimolar doses used.

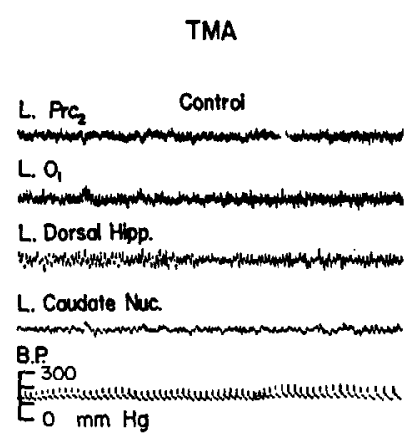

$1.78 \mathrm{mg}$ i.vt.

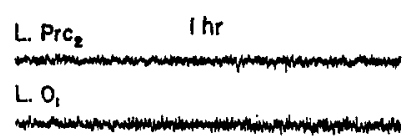

L. Dorsol hipp.

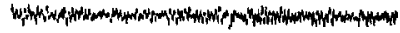

L. Coudate Nuc.

B.P.

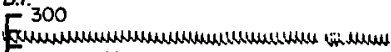

E $0 \mathrm{~mm} \mathrm{Hg}$
$\mathrm{C}_{6}$

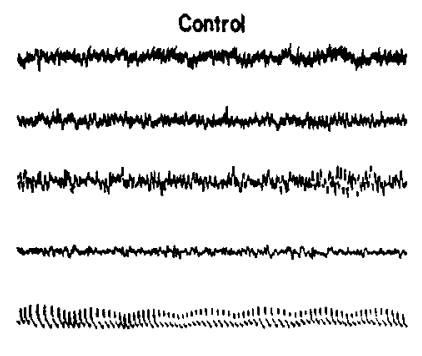

$2.45 \mathrm{mg}$ i.vt.

I hr

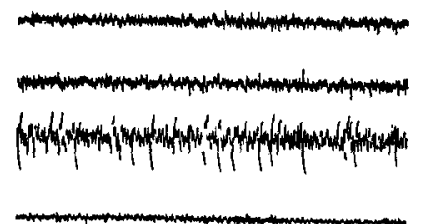

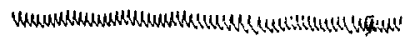

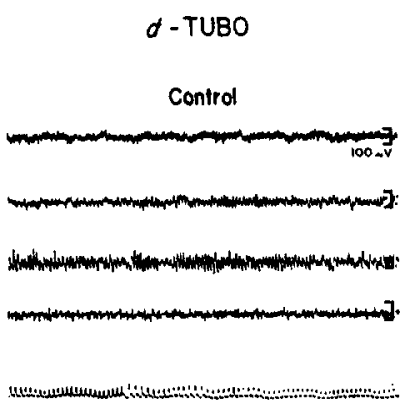

$8.35 \mathrm{mg}$ i.vi.

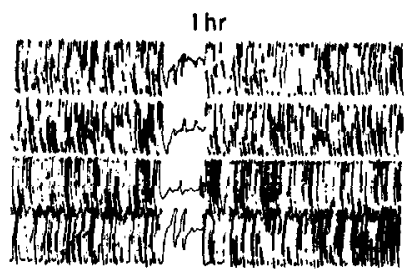

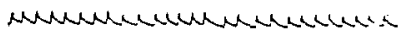

$\stackrel{\sec }{2}$

Fig. 4. FFG effects of varinus quaternary drugs given intraventricularly. Typical EFG effects of various quaternary agents given in equimolar doses to $5 \mathrm{mg}$ of HC-3 are illustrated. Note that the effects of tetramethylammonium (TMA), hexamethonium $\left(\mathrm{C}_{B}\right)$ and $d$-tubocurarine ( $d$-Tubo) are quite different from those of $\mathrm{HC}-3$. 

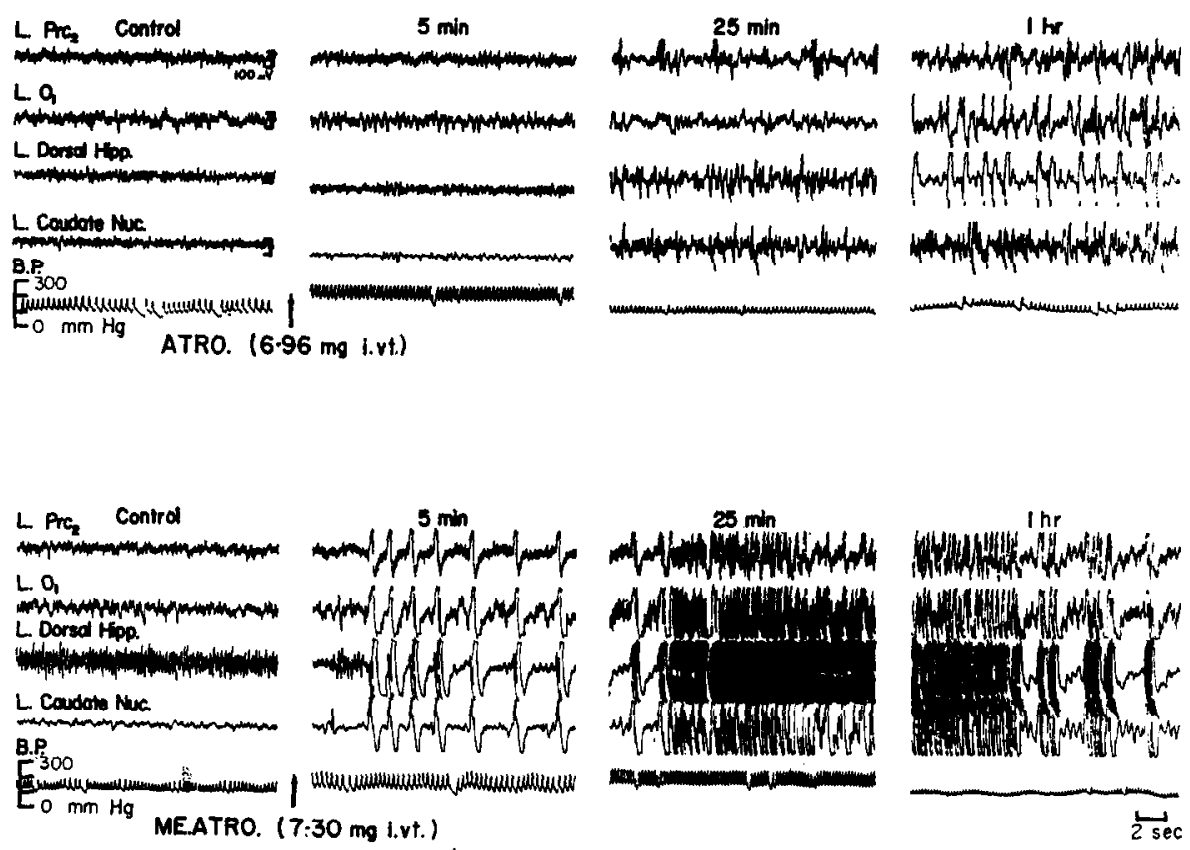

FIG. 5. EEG effects of atropine and methylatropine given intraventricularly. Both drugs were given in twice equimolar doses to $5 \mathrm{mg}$ of $\mathrm{HC}-3$. Note that atropine produces some spiking and slow waves with spindles in $\mathrm{PrC}_{2}$ at $25 \mathrm{~min}$. In contrast methylatropine produces spiking followed by seizures resembling HC-3 seizures.

\section{DISCUSSION}

The results of the present study suggest that the EEG seizures resulting from i.vt. HC-3 probably are not brought about by AChE inhibition, nor are they the result of some non-specific effect of quaternary nitrogen compounds, since TMA failed to elicit seizures at twice equimolar doses. Indeed, the lack of seizures with hexamethonium and atropine indicate that the effect may depend upon the quaternary nitrogen associated with a large cyclic molecule. Furthermore, the differences in the characteristics of the seizures produced by the larger molecules, HC-3, $d$-tubocurarine and methylatropine, seem to indicate a degree of specificity in the site as well as mechanism of action.

Unfortunately we cannot state unequivocally that cholinesterase inhibition does not occur with HC-3 in vivo. HC-3 is a competitive inhibitor (KURTZ et al., 1964) of low affinity and is readily displaced from the enzyme by the substrate. Thus, it would be possible for inhibition to occur in vivo which could not be measured in vitro due to dilution of the HC-3 during homogenization and displacement by the substrate. However, the conclusion that AChE inhibition does not occur to a significant degree can be supported by the observation that the 0.01 equimolar dose of neostigmine, which caused significant inhibition in the periventricular structures, did not consistently cause EEG seizures. In addition, the EEG seizures accompanying AChE inhibition by the equimolar dose of neostigmine were qualitatively very different from the HC-3-induced seizures. Furthermore, the AChlowering effects are the same with $5 \mathrm{mg}$ or $50 \mu \mathrm{g}$ of HC-3 at $4 \mathrm{hr}$ (DREN and Domino, 
1966). It is unlikely that this would be true if the 5-mg dose caused significant AChE inhibition.

Another factor indicating differences in the site or mechanism of action of these drugs is the time lag in the development of HC-3-induced seizure discharge. Full development of seizures usually required 20-30 min with HC-3 and 12-15 min with methylatropine, whereas the $d$-tubocurarine seizures were maximal within $5 \mathrm{~min}$. This time lag, noted with HC-3 in particular, is at variance with the observation of WINTERSTEIN (1961) that the response to i.vt. administered drugs is usually quite rapid. In the case of the response to i.vt. $d$-tubocurarine, Feldberg and FleischHauer (1963) have presented evidence that the primary site of action is the hippocampus.

The time lag in the HC-3 seizure response may be due to either diffusion to a site other than the hippocampus or some cumulative effect with a fairly consistent time constant, or a combination of both. Consistent with the proposal of diffusion was the observation that spiking seemed to occur and become well developed in the caudate nucleus prior to development in the hippocampus. A more thorough investigation of the subcortical response is obviously needed to determine if this is the only site of origin of the EEG seizures and if the lag time is consistent with the time required to reach effective concentrations of $\mathrm{HC}-3$ by diffusion.

The concept of a cumulative effect would seem, at first, to implicate the ACh-lowering effect of HC-3. The data of DREN and Domino (1966) show that, with $50 \mu \mathrm{g}$ i.vt. HC-3 at $1 \mathrm{hr}, \mathrm{ACh}$ levels are somewhat lower in the periventricular structures; however, only the effect on caudate is significant at the 0.05 level (Student's " $t$ " test, group comparison). At this time there is no neocortical EEG effect. The eventual EEG effect of ACh-lowering is a high voltage slow wave pattern, not EEG seizures. In view of this, it seems unlikely that the seizures produced by a high dose of $\mathrm{HC}-3$ are related to ACh-lowering, particularly since the 5-mg dose produces no greater lowering of $\mathrm{ACh}$ at $4 \mathrm{hr}$.

HC-3 could be acting as an excitatory cholinergic agonist, as has been suggested recently by BARROS and DE BALBIAN VERSTER (1966). However, the time lag in onset of activity argues against this. It could also be acting to produce disinhibition by one of two possible mechanisms: by acting as an antagonist of an inhibitory mediator, or by blocking excitation of an inhibitory system. Either of these mechanisms would result in increasing facilitation and recruitment of neurons eventually leading into seizure activity.

In any event, HC-3-induced EEG seizure activity seems to be a direct effect of the drug qualitatively related to cholinergic blockade but differing somewhat from that produced by classic cholinergic blockers.

Acknowledgement-The authors would like to acknowledge the work of Mr. JON FRAPPIER in performing the acetylcholinesterase assays.

\section{REFERENCES}

Adrianov, O. S. and Mering, T. A. (1964), Atlas of the Canine Brain. (English Translation by E. IGNATIEFF; E. F. Dommo, Ed.), Univ. of Michigan, Ann Arbor.

Barros, A. and DE BALBIAN VERSTER, F. (1966). Activation of the central nervous system by hemicholinium. Pharmacologist 8: 184.

DOMER, F. R. and SchurLer, F. W. (1960). Synthesis and metabolic studies of $\mathrm{C}_{14}$-labeled hemicholinium number three. $J$. Am. pharm. Ass. (Sci. Ed.) 49: 553-558.

Domino, E. F., Dren, A. T. and Y AMAMOto, K. (1967). Pharmacologic evidence for cholinergic mechanisms in neocortical and limbic activating systems. Progress in Brain Research, Vol. 28. Elsevier, Amsterdam. (In press). 
Dren, A. T. and Domino, E. F. (1965). Some effects of hemicholinium bromide (HC-3) on EEG desynchronizing mechanisms in the dog. Pharmacologist 7: 143.

Dren, A. T. and DomINo, E. F. (1966). Effects of hemicholinium (HC-3) on EEG activation and brain acetylcholine in the dog. Pharmacologist 8: 183.

Feldberg, W. and FleischHauer, K. (1963). The hippocampus as the site of origin of the seizure discharge produced by tubocurarine acting from the cerebral ventricles. J. Physiol., Land. 168: 435-442.

Feldberg, W., Malcolm, J. L. and Sherwood, S. L. (1956). Some effects of tubocurarine on the electrical activity of the cat's brain. J. Physiol., Lond. 132: 130-145.

GARDINER, J. E. (1961). The inhibition of acetylcholine synthesis in brain by hemicholinium. Biochem. $J$. 81: $297-303$.

Hebi, C. O., Ling, G. M., McGeer, E. G., McGeer, P. L. and Perkins, D. (1964). Effect of locally applied hemicholinium on the acetylcholine content of the caudate nucleus. Nature, Lond. 204: 1309-1311.

Jensen-Holm, J., Lausen, H. H., Mrithers, K. and Moller, K. (1959). Determination of the cholinesterase activity in blood and organs by automatic titration. With some observations on serious errors of the method and remarks on the photometric determination. Acta pharmac. tox. 15: 384-394.

KASE, Y. and BORISON, H. L. (1958). Central respiratory depressant action of "hemicholinium" in the cat. J. Pharmac. exp. Ther. 122: 215-233.

KuRTz, G. S., Baptista los Santos, C. and Romildo, B. J. (1964). Contribution to the pharmacology of hemicholinium, No. 3. Hospital (Rio de J). 66: 9-23.

Lim, R. K. S., LIU, C. and MoffrTt, R. L. (1960). A Stereotaxic Atlas of the Dog's Brain. Thomas, Springfield, Illinois.

SCHUELER, F. W. (1955). A new group of respiratory paralysants. I. The hemicholiniums. J. Pharmac. exp. Ther. 115: 127-134.

Schueler, F. W. (1960). The mechanism of action of hemicholinium. Int. Rev. Neurobiol. 2: 77-97.

VANDER, A. J. (1962). Renal excretion of choline in the dog. Am. J. Physiol. 202: 319-324.

WintersteIn, H. (1961). The action of substances injected into the cerebrospinal fluid and the problems of intracranial receptors. Pharmac. Rev. 13: 71-107. 